\title{
Nonsudden Limits of Heavy-Ion Induced Knockout Reactions
}

\author{
F. Flavigny, ${ }^{1,2}$ A. Obertelli, ${ }^{1}$ A. Bonaccorso, ${ }^{3}$ G. F. Grinyer ${ }^{4}$ C. Louchart, ${ }^{1}$ L. Nalpas, ${ }^{1}$ and A. Signoracci ${ }^{1}$ \\ ${ }^{1}$ CEA, Centre de Saclay, IRFU/Service de Physique Nucléaire, F-91191 Gif-sur-Yvette, France \\ ${ }^{2}$ Instituut voor Kern- en Stralingsfysica, K.U. Leuven, B-3001 Leuven, Belgium \\ ${ }^{3}$ Istituto Nazionale di Fisica Nucleare, Sezione di Pisa, Largo Pontecorvo 3, 56127 Pisa, Italy \\ ${ }^{4}$ National Superconducting Cyclotron Laboratory, Michigan State University, East Lansing, Michigan 48824, USA
}

(Received 1 April 2012; published 20 June 2012)

\begin{abstract}
We report on the single neutron and proton removal reactions from unstable nuclei with large asymmetry $\Delta S=S_{n}-S_{p}$ at incident energies below $80 \mathrm{MeV} /$ nucleon. Strong nonsudden effects are observed in the case of deeply-bound-nucleon removal. The corresponding parallel momentum distributions exhibit an abrupt cutoff at high momentum that corresponds to an energy threshold occurring when the incident energy per particle is of comparable magnitude to the nucleon separation energy. A large lowmomentum tail is related to both dissipative processes and the dynamics of the nucleon removal process. New limits for the applicability of the sudden and eikonal approximations in nucleon knockout are given.
\end{abstract}

DOI: 10.1103/PhysRevLett.108.252501

PACS numbers: $24.50 .+\mathrm{g}, 25.60 . \mathrm{Gc}$

Direct nucleon removal reactions represent a unique way to probe the ground-state wave functions of atomic nuclei. The primary procedure extracts spectroscopic factors from the comparison of experimental data to reaction theory predictions which include the relevant structure information. The concept of spectroscopic factors was first introduced in [1], where its complexity was already discussed. In particular, it was foreseen what has proven to be crucial in the present research with unstable nuclei, namely, the effects of core excitations and correlations. To study ground-state wave functions of short-lived nuclei, onenucleon knockout at intermediate energies in inverse kinematics has been used and has largely contributed to establishing the picture of shell structure away from stability [2]. Shell occupancies and the intrinsic angular momentum of the removed nucleon are extracted from experimental cross sections by comparison to model predictions under the sudden and eikonal (SE) approximation, assuming that the projectile's kinetic energy is large enough to consider its intrinsic degrees of freedom frozen [3]. Time dependent approaches, in which the sudden limit is not applied, exist as well $[4,5]$. The SE approximation is applicable to a quantum mechanical transition when the so-called adiabaticity parameter [6] is small, $\bar{\omega} \ll 1$.

A recent compilation of experimental knockout cross sections at intermediate energies showed a systematic trend when compared to theoretical calculations based on shell-model predictions for shell occupancies and the SE approximations for the nucleon removal reaction [7]: experimental cross sections for the knockout of deeply-bound nucleons were much smaller than theoretical predictions. This unsolved discrepancy still questions our understanding of both nuclear correlations and reaction mechanisms (see, for example, [8-13]).

In the present Letter, we further investigate single nucleon knockout reactions by focusing on light nuclei
${ }^{14} \mathrm{O}$ and ${ }^{16} \mathrm{C}$. Both exhibit a large difference in individual nucleon separation energies, approximately $20 \mathrm{MeV}$, similar to cases where strong discrepancies between experimental and theoretical results have been observed. Both nuclei can be produced with high intensity, allowing a high-statistics momentum distribution to be measured and offering new insight into the reaction mechanism. In this study, incident beam energies below $80 \mathrm{MeV} /$ nucleon were used to investigate the limits of the reaction model and to determine the regime in which spectroscopic information can be reliably extracted.

Two experiments were performed at the National Superconducting Cyclotron Laboratory. In the first, a primary beam of ${ }^{18} \mathrm{O}$ was produced and accelerated to $120 \mathrm{MeV} /$ nucleon by the coupled-cyclotron facility. The ${ }^{16} \mathrm{C}$ secondary beam was produced by fragmentation on a ${ }^{9} \mathrm{Be}$ target at the entrance of the A1900 fragment separator [14] to deliver an almost pure beam (99\%). In the second experiment, an ${ }^{16} \mathrm{O}$ primary beam was fragmented on the same target leading to a secondary beam containing $73(1) \%$ of ${ }^{14} \mathrm{O}$. The ${ }^{16} \mathrm{C}$ and ${ }^{14} \mathrm{O}$ secondary beams impinged on a $188(4) \mathrm{mg} / \mathrm{cm}^{2}{ }^{9} \mathrm{Be}$ target placed at the object point of the S800 spectrograph [15] and surrounded by the SeGA high-purity germanium array [16]. The mid-target energies were 75 and $53 \mathrm{MeV} /$ nucleon, respectively. Time of flight measured upstream of the target provided an unambiguous identification of the incoming particles. The SeGA array was composed of 17 highly-segmented germanium detectors and led to a photopeak efficiency of 2.6(1)\% for a $1 \mathrm{MeV}$ transition. Doppler effect corrections were performed event by event. The projectilelike reaction products were transmitted to the $\mathrm{S} 800$ focal plane. A clear identification of the knockout residues was reached via time of flight between the A1900 and S800 focal planes and energy loss in the ionization chamber. From the coordinates of the residues in the focal plane measured by two 
cathode-readout drift chambers, we reconstructed the outgoing momentum of reaction products and the scattering angles. Thus, the kinematics of the knockout residues were determined and their longitudinal-momentum distributions could be evaluated. The relative momentum spread of ${ }^{16} \mathrm{C}$ and ${ }^{14} \mathrm{O}$ beams on the target was $0.4 \%$ and $0.3 \%$ full width at half maximum, respectively. Despite the S800 large momentum acceptance $( \pm 2.5 \%)$, three different $B \rho$ settings were needed to measure almost complete momentum distributions for each reaction channel.

The one-neutron removal from ${ }^{16} \mathrm{C}$ leads to ${ }^{15} \mathrm{C}$ which has a $5 / 2^{+}$bound excited state at $740 \mathrm{keV} \mathrm{[17]} \mathrm{above} \mathrm{its}$ $1 / 2^{+}$ground state. From the inclusive momentum distribution, shown in Fig. 1(a), we extracted the inclusive cross section of the one-neutron removal to be $81(7) \mathrm{mb}$. The error bars contain uncertainties on beam intensity $(5.5 \%)$, particle selection (3\%), target thickness (2\%), background subtraction (2\%), and acceptance corrections (4\%) added quadratically to the statistical uncertainty. The SE approximation as used in [7] predicts $89 \mathrm{mb}$ (see Table I), in good agreement. The single-particle cross sections were calculated using core densities obtained from Hartree-Fock (HF) calculations with the Sly4 interaction [19]. In the case of the neutron removal from ${ }^{16} \mathrm{C}$, the neutron $1 d$ and $2 s$

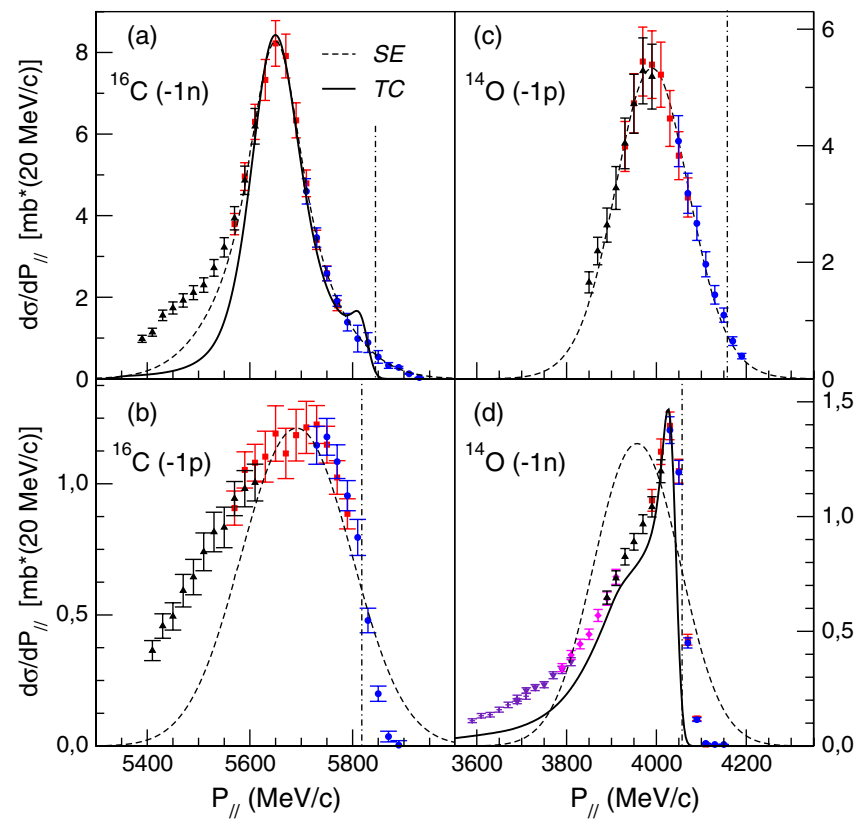

FIG. 1 (color online). Parallel-momentum distributions of projectilelike residues from inclusive one-nucleon knockout: (a) ${ }^{15} \mathrm{C}$ and (b) ${ }^{15} \mathrm{~B}$ from ${ }^{16} \mathrm{C}$, (c) ${ }^{13} \mathrm{~N}$ and (d) ${ }^{13} \mathrm{O}$ from ${ }^{14} \mathrm{O}$. Colors represent different settings for the $\mathrm{S} 800$ spectrometer. Calculated parallel-momentum distributions at mid-target energies convoluted with the momentum profile of the beam and shifted by a momentum (typically $100 \mathrm{MeV} / c$ ) corresponding to the residue's energy loss in half of the target are shown as SE (dashed line) and TC (solid line) (see text). Theoretical distributions have been normalized to the data. Cutoff positions according to Eq. (1) are shown as vertical lines. single-particle wave functions were calculated in a WoodsSaxon potential whose radius and depth were taken from [20]. Spectroscopic factors calculated with the WBT shellmodel interaction [18] in the $p s d$ valence space (limited to two $\hbar \omega$ excitations) were used. Theoretical parallelmomentum $\left(P_{\|}\right)$distributions from the SE approximation have been calculated for the removal of both $\ell=2\left(5 / 2^{+}\right)$ and $\ell=0\left(1 / 2^{+}\right)$nucleons, following the derivation of [21]. Their peak amplitudes have been adjusted to the inclusive momentum distribution (dashed lines in Fig. 1). The position of their maximum has been set to the expected mean momentum of the residue at the exit of the target, taking into account energy loss and $Q$ values. Very good agreement is found with the exception of a tail at low momentum leading to a slight asymmetry as already observed, for example, in [22-25]. The populations of the bound excited state and the ground state contribute to 56 (5)\% and 44(5)\% of the inclusive cross section, respectively, in agreement with previous studies [20,22,26].

The ${ }^{16} \mathrm{C}\left({ }^{9} \mathrm{Be}, X\right){ }^{15} \mathrm{~B}$ reaction requires the removal of a deeply-bound proton with $S_{p}=22.6 \mathrm{MeV}\left(S_{n}=\right.$ $4.25 \mathrm{MeV})$. The spectroscopy of ${ }^{15} \mathrm{~B}$ has been previously performed [27] and shows a $3 / 2^{-}$ground state and two bound excited states: a $7 / 2^{-}$state at $2734 \mathrm{keV}$ and a $5 / 2^{-}$ state at $1327 \mathrm{keV}$ above the ground state. The spin and parity of these states, consistent with the observed decay scheme, rely on the comparison with shell-model predictions using the WBT interaction. We measured an inclusive cross section of 20(2) mb. The occurrence of effects beyond the SE approximation is visible on the $P_{\|}$distribution shown in Fig. 1(b): the momentum distribution is asymmetric and displays a very large tail at low momentum. The spectrum of prompt deexcitation gamma rays measured in coincidence with ${ }^{15} \mathrm{~B}$ is shown in Fig. 2 . The cross section extracted for the population of the ground state is $18(2) \mathrm{mb}$, much lower than the theoretical prediction of $50 \mathrm{mb}$ assuming $\ell=1$ removal. The population of the excited states is $1.3(2) \mathrm{mb}$ for the $5 / 2^{-}$state and $0.8(1) \mathrm{mb}$ for the $7 / 2^{-}$ state. In the case of a direct process, the population of these states is not expected. Indeed, assuming that protons are contained inside the large spsd valence space, the only negative-parity states that can be populated come from $\ell=1$ removal and are, therefore, limited to $J^{\pi}=1 / 2^{-}$ or $3 / 2^{-}$. The $P_{\|}$distributions for the direct population of the ${ }^{15} \mathrm{~B}$ states have been extracted from the coincidence with photopeak events (see inset in Fig. 2) by subtracting eventual feeding from upper states. The distributions for excited states are peaked at momentum values of $\sim 5500-5650 \mathrm{MeV} / c$, well below the maximum of the ground-state distribution, demonstrating that a large part of the population results from dissipative processes during the core-target interaction time. Strong asymmetries in the fragmentation of intermediate energy stable beams have long been known (for example, see [28]) and attributed to dissipative processes. In the case of exotic beams, clear proof of 
TABLE I. Summary of one-nucleon knockout results from ${ }^{14} \mathrm{O}$ at $53 \mathrm{MeV} /$ nucleon and ${ }^{16} \mathrm{C}$ at $75 \mathrm{MeV} /$ nucleon. We show inclusive cross sections $\sigma_{\mathrm{SE}}$ from the sudden and eikonal approach and $\sigma_{\mathrm{TC}}$ from the transfer to the continuum approach and the measured $\left(\sigma_{\text {exp }}\right)$ cross sections. Spectroscopic factors $C^{2} S$ are calculated with the WBT interaction in the $p s d$ valence space [18].

\begin{tabular}{|c|c|c|c|c|c|c|c|}
\hline Beam & Res. & $E(\mathrm{MeV})$ & $J^{\pi}$ & $\sigma_{\exp }(\mathrm{mb})$ & $C^{2} S$ & $\sigma_{\mathrm{SE}}(\mathrm{mb})$ & $\sigma_{\mathrm{TC}}(\mathrm{mb})$ \\
\hline${ }^{14} \mathrm{O}$ & ${ }^{13} \mathrm{~N}$ & 0.0 & $1 / 2^{-}$ & $58(4)$ & 1.55 & 55 & not applicable \\
\hline${ }^{14} \mathrm{O}$ & ${ }^{13} \mathrm{O}$ & 0.0 & $3 / 2^{-}$ & $14(1)$ & 3.15 & 54 & 34 \\
\hline \multirow[t]{3}{*}{${ }^{16} \mathrm{C}$} & ${ }^{15} \mathrm{~B}$ & 0.0 & $3 / 2^{-}$ & $18(2)$ & 2.95 & 50 & not applicable \\
\hline & & 1.33 & $\left(5 / 2^{-}\right)$ & $1.3(2)$ & $\cdots$ & $\cdots$ & $\cdots$ \\
\hline & & 2.73 & $\left(7 / 2^{-}\right)$ & $0.8(1)$ & $\cdots$ & $\cdots$ & $\cdots$ \\
\hline \multirow[t]{3}{*}{${ }^{16} \mathrm{C}$} & ${ }^{15} \mathrm{C}$ & 0.0 & $1 / 2^{+}$ & $36(5)$ & 0.89 & 60 & 59 \\
\hline & & 0.74 & $5 / 2^{+}$ & $46(6)$ & 0.90 & 30 & 31 \\
\hline & & total & & $81(7)$ & & 90 & 90 \\
\hline
\end{tabular}

this has been found in a very exclusive measurement at GANIL for ${ }^{6} \mathrm{He}$ [29], while a broadening of the transverse momenta for the tails of core longitudinal distributions [23] and energy damping in Si telescope measurements [24] have been identified with such processes. In addition, the highmomentum part of the distribution shows a sharp edge, indicating that the SE approximation is not appropriate for the present reaction channel at $75 \mathrm{MeV} /$ nucleon. For illustration, Fig. 1(b) shows a typical momentum distribution from a sudden process in the SE limit.

Nucleon removal from ${ }^{14} \mathrm{O}\left[S_{n(p)}=23.2(4.63) \mathrm{MeV}\right]$ at $53 \mathrm{MeV} /$ nucleon results in similar conclusions. Neither ${ }^{13} \mathrm{O}$ nor ${ }^{13} \mathrm{~N}$ exhibit bound excited states. The momentum distribution [see Fig. 1(c)] is symmetric and in good agreement with predictions assuming $\ell=1$ removal. The oneneutron removal cross section is measured to be 14(1) mb, $26 \%$ of the expected $54 \mathrm{mb}$ prediction. A very asymmetric momentum distribution is observed [Fig. 1(d)], showing a sharp high-energy edge, more pronounced than the ${ }^{15} \mathrm{~B}$ case and strikingly different from the symmetric distributions

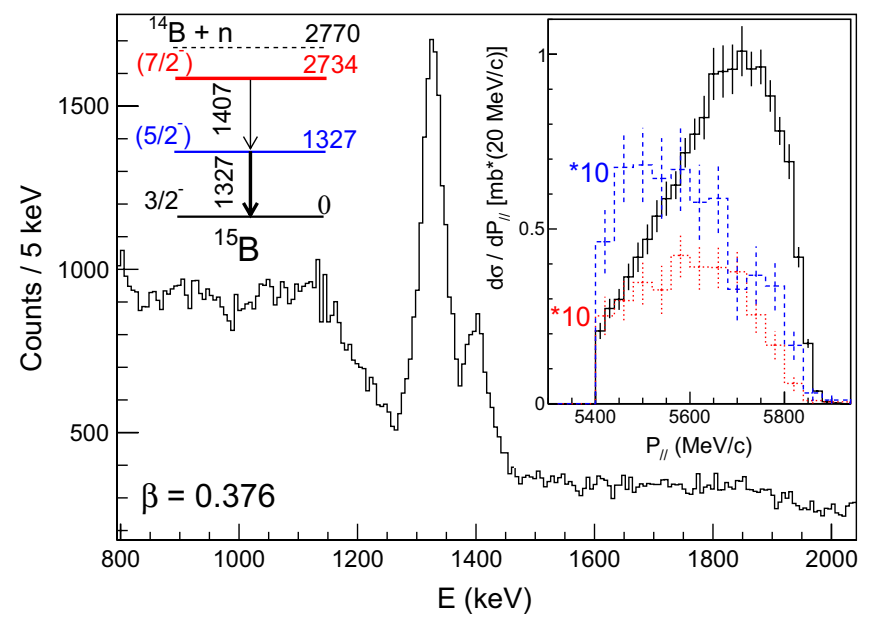

FIG. 2 (color online). Doppler-corrected gamma spectrum measured in coincidence with ${ }^{15} \mathrm{~B}$ from ${ }^{16} \mathrm{C}\left({ }^{9} \mathrm{Be}, X\right){ }^{15} \mathrm{~B}$ at $75 \mathrm{MeV} /$ nucleon. (inset) Parallel-momentum distribution for the population of the ground state (continuous line, black), the first (dashed line, blue), and the second excited state (dotted line, red). that are usually observed at higher incident energies where the SE approximation is valid.

In the two cases of neutron breakup studied in this work, the dimensionless adiabaticity parameter $\bar{\omega}$ is evaluated as 2.25 and 0.24 for ${ }^{14} \mathrm{O}$ and ${ }^{16} \mathrm{C}$ projectiles, respectively. As a result, the SE approximation is only valid for the ${ }^{16} \mathrm{C}$ projectile. When this approximation is not valid, we interpret the momentum distributions from an alternative theoretical approach: the transfer to the continuum (TC) method [30,31]. TC is restricted to neutron removal because of the use of Hankel functions for the initial state, which allows the removed neutron energy distribution in the continuum to be calculated analytically via a timedependent transition amplitude. As such, it contains energy and momentum conservation between the partners of the reaction, and the corresponding parallel momentum of the ejectile is obtained from

$$
P_{\|}=\sqrt{\left(T_{p}-S_{n}-\varepsilon_{f}\right)^{2}+2 M_{r}\left(T_{p}-S_{n}-\varepsilon_{f}\right)},
$$

where $M_{r}$ is the mass of the residue, $T_{p}$ is the initial kinetic energy of the projectile, and $S_{n}$ is the nucleon separation energy from the initial bound state. The neutron final continuum energy with respect to the target is given by $\varepsilon_{f}$. Equation (1), together with the appropriate Jacobian, enables the conversion from the final neutron energy spectrum to the core final momentum spectrum. The threshold at $\varepsilon_{f}=0$ is the same for stripping and diffraction, when the core-target interaction is considered elastic. While Eq. (1) has a general validity and could be introduced independently from the reaction model in order to calculate the breakup threshold, a microscopic calculation of the $\varepsilon_{f}$ distribution allows a consistent description of both the threshold effect and the full $P_{\|}$distribution. The momentum thresholds in the one-nucleon removal, after correction of energy loss in the target, are predicted to be at $P_{\|}=5846,5821,4161$, and $4059 \mathrm{MeV} / c$ for ${ }^{16} \mathrm{C}(-1 n)$, ${ }^{16} \mathrm{C}(-1 p),{ }^{14} \mathrm{O}(-1 p)$, and ${ }^{14} \mathrm{O}(-1 n)$, respectively. These momentum cutoffs are in perfect agreement with what is observed experimentally for deeply-bound nucleon removal (see Fig. 1). For the neutron- ${ }^{9} \mathrm{~B}$ target interaction, 
a new parameterization has been used which reproduces also the data at $E_{\text {inc }}<20 \mathrm{MeV}$. Further details of the method can be found in previous references. In the present calculation, the core-target $S$ matrices are calculated from Hartree-Fock densities within an eikonal approach. The initial bound state wave functions for ${ }^{16} \mathrm{C}$ are the same as in the previous eikonal calculation and for ${ }^{14} \mathrm{O}$ by using a neutron single particle wave function calculated in a Woods-Saxon potential with depth $60 \mathrm{MeV}, r_{0}=$ $1.40 \mathrm{fm}$, and $a=0.7 \mathrm{fm}$ with a spin-orbit potential with depth $V_{\text {so }}=5.5 \mathrm{MeV}$ to reproduce both the experimental separation energy $S_{n}=23.2 \mathrm{MeV}$ and the theoretical radius of the neutron wave function as calculated within the HF formalism. The resulting momentum distributions are shown in Fig. 1. The $P_{\|}$distribution for neutron removal from ${ }^{16} \mathrm{C}$ is in good agreement with experimental data, and the distribution is similar to that of eikonal predictions, giving similar contributions for the $s$ state and $d$ state. Total breakup cross sections are given in Table I. Interestingly, the loosely-bound nucleon removal cross sections from the SE and TC models are the same, whereas in the case of the one-neutron removal from ${ }^{14} \mathrm{O}$ the TC model gives a $30 \%$ smaller cross section than the SE calculation. The small bump close to threshold originates from the population of a $d_{5 / 2}$ resonance state present in the $n-{ }^{9} \mathrm{Be}$ continuum around $3 \mathrm{MeV}$, not observed in the data. The calculated distribution for the deeply-bound neutron removal from ${ }^{14} \mathrm{O}$ reproduces very well the high-energy cutoff due to the threshold effect resulting from Eq. (1), as well as the position of the maximum and the width of the momentum distribution. The shoulder at $3900 \mathrm{MeV} / c$ in the calculated $P_{\|}$distribution, barely visible in the data, comes from both the intrinsic momentum distribution of the removed nucleon and the energy dependence of the $n-{ }^{9} \mathrm{Be}$ interaction cross section. Interestingly, the TC approach predicts also a rather large low-momentum tail for the distribution. It is the first time a microscopic calculation predicts such asymmetric momentum distributions in the case of deeplybound nucleon removal. Although the dynamics of the reaction cannot be reproduced exactly by the present analysis, it suggests a link between the removed-nucleon dynamics and the low-momentum tail of the heavy residue, in addition to the dissipative processes indicated earlier.

The present study shows a very strong asymmetry of the $P_{\|}$distribution when the initial neutron separation energy and the beam incident energy per particle have comparable magnitude $[4,24,32]$. The concept of kinematical limit is not a new one but was already discussed at the time of stable beam studies some three decades ago [28,33] and for exotic beams in $[4,32]$. This effect is enhanced in the present case due to the strong increase of the free-neutron- ${ }^{9} \mathrm{Be}$ cross section at the low energies which dominate the dynamics of the reaction. In Fig. 3, several experiments from NSCL $[7,34]$ and GANIL $[22,23]$ are gathered as a function of the beam energy and the separation energy of the removed

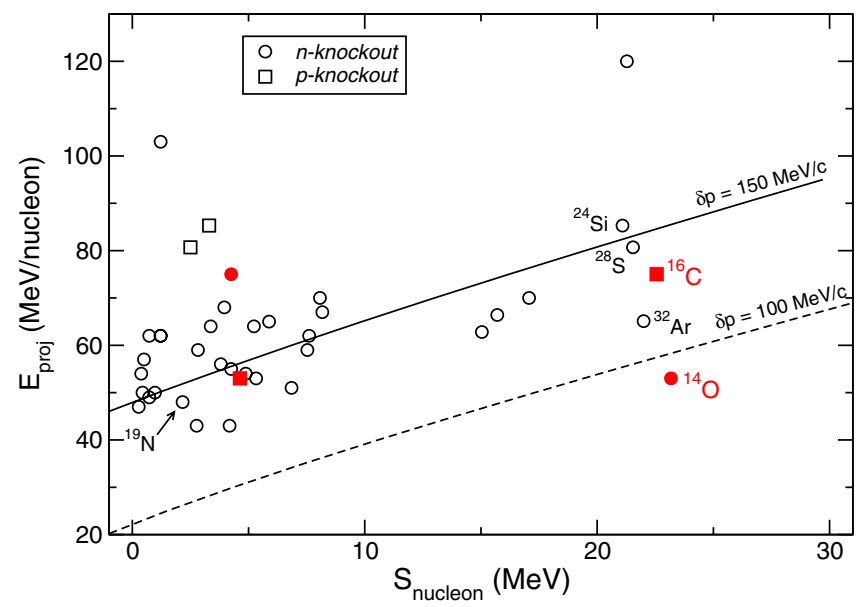

FIG. 3 (color online). Nucleon-removal experiments from the literature $[7,22,34]$ plotted as a function of the energy per nucleon of the projectile and the separation energy of the removed nucleon. The lines correspond to cutoffs appearing at $\delta_{p}=100$ and $150 \mathrm{MeV} / c$ with respect to the center of the SE distribution. Data from the present experiment are shown as filled markers.

nucleon. It appears that several of these previous measurements may have been affected by this threshold effect. Below the $100 \mathrm{MeV} / c$ line, the experimental momentum distributions are strongly deformed by the presence of the kinematical cutoff as observed in the ${ }^{14} \mathrm{O}(-1 n)$ reaction. Edge effects also occur in several data sets beyond this line, namely, the present ${ }^{16} \mathrm{C}(-1 p)$ channel but also data from previous measurements with incident energy below $85 \mathrm{MeV} /$ nucleon, such as ${ }^{28} \mathrm{~S},{ }^{24} \mathrm{Si}$ [7], and ${ }^{16} \mathrm{C},{ }^{19} \mathrm{~N}$ [23]. In Ref. [23], where the one neutron removal from ${ }^{16} \mathrm{C}$ at $55 \mathrm{MeV} /$ nucleon is reported, a cutoff is visible as predicted from Fig. 3, whereas in the present measurement at $75 \mathrm{MeV} /$ nucleon no cutoff is seen, as expected. In the present case of ${ }^{14} \mathrm{O}(-1 n)\left[{ }^{16} \mathrm{C}(-1 p)\right]$, the high-momentum cutoff, shown as vertical lines in Fig. 1, represents $22 \%$ (14\%) of the $P_{\|}$distribution from SE approximation.

In summary, the single nucleon removal cross sections and the corresponding detailed parallel-momentum distributions were measured for light nuclei with large $\Delta S \sim 20 \mathrm{MeV}$ at incident energies below $80 \mathrm{MeV} /$ nucleon. The parallelmomentum distributions obtained from the removal of a deeply-bound nucleon from the studied nuclei exhibit (i) a large tail at low momentum originating in part from dissipative processes during the core-target interaction and (ii) an abrupt cutoff at high momentum, expressed as a function of the energy of the projectile and the separation energy of the removed nucleon. In this study, we define a limit of the energetic regime one can use to safely extract structure information on the projectile by comparing the experimental shape of the parallel-momentum distribution to predictions from standard sudden and eikonal approaches. For incident energies of comparable magnitude to the initial neutron separation energy, the final state interaction of the neutron with the target distorts such distributions, while energy 
conservation conditions restrain some part of it from being visible in the final state. Core excitations in the nucleonremoval reaction have to be further investigated to control the reliability of spectroscopic information extracted from wellbound nucleon removal.

The authors are grateful to the S800 and gamma groups of the NSCL for their essential support in the collection of data. D. Bazin, B. A. Brown, A. Gade, W. Mittig, and J. Tostevin are deeply thanked for fruitful discussions.

[1] M. H. Macfarlane and J. B. French, Rev. Mod. Phys. 32, 567 (1960).

[2] P. G. Hansen and J. A. Tostevin, Annu. Rev. Nucl. Part. Sci. 53, 219 (2003).

[3] R. J. Glauber, in Lecture Notes in Theoretical Physics, edited by W.E. Britten and L. G. Dunham (Interscience, New York, 1958), Vol. 1, p. 315-414.

[4] A. Bonaccorso, Phys. Rev. C 60, 054604 (1999).

[5] H. Esbensen and G. F. Bertsch, Phys. Rev. C 64, 014608 (2001).

[6] A. Winther and K. Adler, Nucl. Phys. A319, 518 (1979).

[7] A. Gade et al., Phys. Rev. C 77, 044306 (2008).

[8] J. Lee et al., Phys. Rev. Lett. 104, 112701 (2010).

[9] C. Barbieri, Phys. Rev. Lett. 103, 202502 (2009).

[10] F. Flavigny, A. Obertelli, and I. Vidana, Phys. Rev. C 79, 064617 (2009).

[11] N. K. Timofeyuk, Phys. Rev. Lett. 103, 242501 (2009).

[12] Ø. Jensen, G. Hagen, M. Hjorth-Jensen, B. A. Brown, and A. Gade, Phys. Rev. Lett. 107, 032501 (2011).

[13] C. Louchart, A. Obertelli, A. Boudard, and F. Flavigny, Phys. Rev. C 83, 011601 (2011).

[14] D. J. Morrissey B.M. Sherrill, M. Steiner, A. Stolz, andI. Wiedenhoever, Nucl. Instrum. Methods Phys. Res. Sect. B 204, 90 (2003).
[15] D. Bazin, J.A. Caggiano, B.M. Sherrill, J. Yurkon, and A. Zeller, Nucl. Instrum. Methods Phys. Res. Sect. B 204, 629 (2003).

[16] W.F. Mueller, J.A. Churcha, T. Glasmachera, D. Gutknechtc, G. Hackmana, P.G. Hansena, Z. Hua, K.L. Millera, and P. Quirinc, Nucl. Instrum. Methods Phys. Res. Sect. A 466, 492 (2001).

[17] F. Ajzenberg-Selove, Nucl. Phys. A523, 1 (1991).

[18] E. K. Warburton and B. A. Brown, Phys. Rev. C 46, 923 (1992).

[19] E. Chabanat, P. Bonche, P. Haenselc, J. Meyer, and R. Schaeffer, Nucl. Phys. A627, 710 (1997).

[20] V. Maddalena et al., Phys. Rev. C 63, 024613 (2001).

[21] C. A. Bertulani and P.-G. Hansen, Phys. Rev. C 70, 034609 (2004).

[22] E. Sauvan et al., Phys. Lett. B 491, 1 (2000).

[23] E. Sauvan et al., Phys. Rev. C 69, 044603 (2004).

[24] F. Negoita et al., Phys. Rev. C 59, 2082 (1999).

[25] J. A. Tostevin, D. Bazin, B. A. Brown, T. Glasmacher, P. G. Hansen, V. Maddalena, A. Navin, and B. M. Sherrill, Phys. Rev. C 66, 024607 (2002).

[26] T. Yamaguchi et al., Nucl. Phys. A724, 3 (2003).

[27] M. Stanoiu et al., Eur. Phys. J. A 22, 5 (2004).

[28] Y. Blumenfeld, Ph. Chomaz, N. Frascaria, J. P. Garron, J. C. Jacmart, and J. C. Roynette, Nucl. Phys. A455, 357 (1986).

[29] Y. Périer, B. Lotta, J. Galin, E. Liénard, M. Morjean, N. A. Orr, A. Péghaire, B. M. Quednau, and A.C.C. Villari, Phys. Lett. B 459, 55 (1999).

[30] A. Bonaccorso and D. M. Brink, Phys. Rev. C 38, 1776 (1988).

[31] A. Bonaccorso and D. M. Brink, Phys. Rev. C 44, 1559 (1991).

[32] A. Bonaccorso and G. F. Bertsch, Phys. Rev. C 63, 044604 (2001).

[33] T. Fujita and J. Hüfner, Nucl. Phys. A343, 493 (1980).

[34] G. F. Grinyer et al., Phys. Rev. Lett. 106, 162502 (2011). 